\title{
TEMPORAL CONSISTENCY AMONG FIVE-SENSORY EVENTS: APPLICATION TO MULTIMEDIA TECHNOLOGY
}

\author{
V.K.Murthy \\ edayathuk@gmail.com \\ E.V.Krishnamurthy \\ Australian National University, Canberra, Australia \\ Evk.Krishnamurthyeanu.edu.au
}

\begin{abstract}
While transmitting multi or five- sensory information after transduction, the concept of temporal consistency becomes an important issue at the receiving end from the point of view of context. Any violation of temporal consistency among the different decoded sensory information can lead to a failure of context, thereby making the multisensory system ineffective in creating unified percept and bringing in reality to any scene. Therefore, when a multisensory information is captured, transduced and the resulting convergent (fused or mashed) information is transmitted as a multimedia information, and again reverse transduced and interpreted at the receiving end, the temporal constraints among the different sensory responses need to be consistent and preserved. Since a multimedia message containing fused five-sensory information describes patterns of occurrence of events in the real world, the invocation of a partially ordered sequence of such messages in a given context should take us from one consistent state to another consistent state and checking for context violations. In this paper we describe a cooperating-agents based algorithm to detect temporal consistency among the five sensory events and for multimedia five-sensory processing. The theoretical formulation requires elementary principles of temporal logic. The cooperative aspects of the agent-based algorithm is illustrated using consistent and inconsistent examples and the UML activity diagram. This algorithm will be very useful for applications in Web 2.0 location-based context awareness in multimedia technology.
\end{abstract}

\section{KEY WORDS}

Agent, AmI (ambient intelligence computing), Choreography, Context awareness, Distributed context-aware cognitive system, Five - sensory multimedia technology, Orchestration, Proto-cols, Temporal consistency, UML activity diagram, Web 2.0.

\section{INTRODUCTION}

THE ultimate goal of the Ambient Intelligent computing environment (AmI) consisting of peers (agents or other intelligent devices), Remagnino et al., [1] is to realize in- silico consciousnessthat leads towards omniscient computation in which a problem is solved collaboratively in a distributed manner by communication though sensory mechanisms evoking conscious response, Baars [2], [3], Blackmore, [4], Edelman, [5], Murthy and Krishnamurthy [6]. The evolution of Wireless Broad band and Web 2.0 providing web services from syntactic to semantic and presently to a pragmatic (user-conscious) level, de Bruign et al [7], has further strengthened the AmI environment. Also the recent innovative studies by Chalmers et al [8], [9], Kim et al [10], [11] on five senses multimedia technology- in which five sensory messages- visual (eyes), aural (ears), haptic (touch), smell (nose) and taste (tongue) are perceived, as if they are real 
have considerably elevated the status of web-services along with AmI, as an integral part of human consciousness. This is due to the capacity of the brain in coordinating the different sensory signals arising from a common source, thereby providing us with a unified percept of the world.

While transmitting multi or five- sensory information after transduction, the concept of temporal consistency becomes an important issue at the receiving end from the point of view of context. Any violation of temporal consistency among the different decoded sensory information can lead to a failure of context, thereby making the whole AmI system ineffective. Outstanding cognitive psychologists now agree, that consciousness and context are inseparable twin issues in creating unified percept and bringing in reality to any scene. Therefore, when a multisensory information is captured, transduced and the resulting convergent (fused or mashed) information is transmitted as a multimedia information, and again reverse -transduced and interpreted at the receiving end, the temporal constraints among the different sensory responses need to be consistent and preserved. Since a multimedia message containing fused five-sensory information describes patterns of occurrence of events in the real world, the invocation of a partially ordered sequence of such messages in a given context should take us from one consistent state to another consistent state and checking for context violations. This corresponds to monitoring the action of an organism in its environment through an internal and environmental feedback. In animals, any failure of this monitoring action results in brain disorder, creating a false response, unable to recognize its falsity, and check its failure, namely, its inappropriateness in a context, Hirstein, [12]. Detecting context violations is carried out by the hippocampus in the brain, to spot events that violate our expectations and triggering attention mechanisms to direct the surprising events to our consciousness. If conflicting information is present in different sensory modalities, then our perception of events may be degraded or altered in ways that reflect a synthesis of different sensory cues that can be catastrophic, Calvert et al. [13], Spence and Driver [14].

Further, in AmI, not only the context, but also the expectation and intention play a crucial role. In expectation, we await classes of inputs rather than a specific input so that one can be prepared for serious violations that have to be resolved. Also one may end up with unexpected events resulting in the violation of expectations. Intention is a state prior to an action. Baars [2],[3], identifies it as a goal context. Since a voluntary action follows an intention, a conscious system looks for an action that is consistent and failure-free with one's own goals; this is a built- in feature in animal brains.

In real-life applications, intention, context, action and expectation, are interleaved and can enable, disable (inhibit) each other or remain dormant. To have a successful implementation of five sensory AmI, we need to have a suitable set of rules (protocols) to deal with IntentionContext-Action. This set of rules specifies the required precondition / post condition for an appropriate action and is highly problem domain dependent. Such a precondition or post condition can be a two-valued logical predicate (true or false), or a fuzzy or probabilistic measure arising from experience, Murthy and Krishnamurthy [6].

\section{BACKGROUND}

A sequence of such multi-sensory events in a multi-media environment appears real, as long as there are no context violations. This means the web-service provider should choreograph the events occurring at the provider (transmitter) -end in the web-service, and orchestrate the same events at the requestor-side faithfully, see de Bruign et al [7]. Thus we are considering a world as an entity that changes with time and at each point of time the world is in one particular state and the perceiver's view changes moment to moment introducing new contexts and new events. In order to provide such a web-service, we need to devise a software construct that can maintain temporal consistency among events and detect context violations arising due to the uncertain nature of connectivity of ubiquitous devices and networks, and the failure of the processes involved in interactions among devices, in an unpredictable environment. This is illustrated by the following simple example. 
Consider the following scenario in which a commercial movie clip has five-sensory multimedia contents transmitted over Web 2.0. The clip may present a visual scene in the making of a particular Peruvian truffle (Peruvian chocolate) -having a definite flavor (taste and smell), Goldstein [15]. When a chef constitutes a mixture of the required ingredients, apart from the associated sounds and imagery, the smell and flavor fill the environment. The truffle is then made, and the students present in the scene enjoy its flavor and taste! This sequence of events is partially ordered in time and the transduction of these signals takes varying time. In order to preserve the context we need to ensure that the temporal consistency is not violated. For example we do not want the flavor of the truffle to appear at the receiving end, even before the mixture is made or after it has been eaten. This consistency is analogous to the "lip synchronization problem (the synchronization of a video with audio and lip movements)". When video and audio signals are transported via different facilities (e.g., wireless link and a landline) that have significantly different times, it is necessary to delay the earlier of the two signals electronically to allow for the difference in times. Similar approaches may be needed for other five-sensory signals to verify temporal consistency, if a failure is detected. This means we should have a complete knowledge of the cognitive speed of human sensory transduction, involving memory, relaxation, response and processing time, decay time as well as how the relative speeds of the transmission of electronic, chemical and pressure signals can interfere with the context. These aspects are beyond the scope of this chapter, and currently form a major area of study in humancomputer interaction, sensory psychology and biology, Calvert et al [13], Spence and Driver [14]. In this paper we will only be concerned with checking of temporal consistency among the different events and its failure.

\section{FOCUS OF THE PAPER}

\subsection{Issues}

The main purpose of this paper is to devise the basic software construct needed for checking for temporal consistency among the sensory events in a given spatial location. The goal of temporal reasoning is to validate the different propositions involving time and evaluate the consistency among these statements, using the various local constraints that can be either qualitative or quantitative, and arriving at a globally consistent picture of the whole scenario. This is a computationally hard problem, in general. However, if we restrict ourselves to a problem, in which any two events are related by a single temporal constraint involving positive integer inequalities (by a suitable choice of time units), then the problem is solvable in polynomial time. If there are many constraints among events, the problem needs to be broken into pieces involving the possible combinations among the various constraints. This leads to an exponential growth in complexity. In this paper, we will only deal with the simple temporal consistency problem among a set of agents. For this purpose we need to assume that the agents are using the same accurate clock and the constraints are exact.

In the first two sections below, we describe the qualitative and quantitative temporal consistency problem. Following this, the next section describes the representation of the quantitative temporal consistency problem as a Directed graph. Here, we describe the basic directed graph representation of temporal constraints among events, a node representing an event. The application of a simple yet powerful theorem by Shostak, [16] is described in this section.. We then describe the cooperative multi-agent paradigm for detecting the global temporal consistency. The agent-based detection of temporal consistency is illustrated through an UML activity diagram. Finally we describe the future trends and provide the concluding remarks.

\section{TEMPORAL CONSISTENCY}

Given a set of sensory events, satisfying certain time constraints, the temporal consistency problem deals with reasoning of this set of events and evaluate whether the set is consistent or 
conflict-free. We assume that we are dealing with real-life macro-events and not cosmic or subatomic events and time has a beginning and has an arrow towards the future. We consider a simple set of constraints in which any two events are related by a single constraint in a welldefined range.

\subsection{Events}

Events are uniquely labelled and ordered thus: $\mathrm{E}(0), \mathrm{E}(1), \mathrm{E}(2), \ldots \mathrm{E}(\mathrm{i}), \mathrm{E}(\mathrm{i}+1), \ldots .$. $E(j), \ldots$, where we assume that $E(0)$ is the standard reference time point or the beginning of the world we consider .E(i) is before $\mathrm{E}(\mathrm{i}+1)$ is denoted by $\mathrm{E}(\mathrm{i})<\mathrm{E}(\mathrm{i}+1)$. Accordingly we assume that the events belong to a well-founded set, defined below.

\subsection{Well-founded Sets}

A binary relation < is well-founded over a class of objects, if it satisfies the no-decreasing condition; that is, there is no infinite sequence of objects decreasing with respect to that relation. In other words, there are no infinite sequences of objects: $\{x(0), x(1), x(2), x(3), \ldots$. such that:

$x(0)>x(1)>x(2)>x(3)>\ldots$ where $>$ is the inverse binary relation of $<$.

For a detailed study of well-founded sets and relations, and related temporal logic, see Manna and Waldinger [17]. A set in which the elements are related through a well-founded relation is called a well-founded set.

\section{QUALITATIVE CONSISTENCY}

We use the terminology of Apt, [18], Dechter [19], to describe the qualitative temporal consistency problem and then extend it to the quantitative temporal consistency problem.

We assume that every event $\mathrm{A}$ has a trigger-beginning $\mathrm{Ab}$ and a trigger-end $\mathrm{Ae}$, and the duration of an event is the time interval in integral units, between these two well-defined points. This means also for real-world events $\mathrm{Ab}$ occurs before $\mathrm{Ae}$, or $\mathrm{Ab}<\mathrm{Ae}$ and $\mathrm{Ab}=\mathrm{Ae}$, if and only if, the event is a trigger event. The trigger events are like impulse (delta) functions having no width. This approach helps us to extend the qualitative temporal consistency problem to quantitative temporal consistency.

We denote the following seven temporal relations, Apt [18], Dechter, [19]:

before, meets, overlaps, starts, during (contained in), finishes, equals

in the above notation.

Note that we are permitted to use only the relation $<$ to preserve the well-founded relation; however, we will write $\mathrm{x}=\mathrm{y}$, if and only, if $\mathrm{x}<\mathrm{y}$ and $\mathrm{y}<\mathrm{x}$ meaning that $\mathrm{x}$ and $\mathrm{y}$ are coincident.

Also consider the seven " inverse relations :

after, met by, overlapped by, started by, contained by, finished by, equals.

These relations are grammatical transformations from the active to passive voice in English, and can equally well be represented by analogous relations.

The following qualitative inequalities define the seven temporal relations :

0 . For every event: $A b<A e$, and $A b=A e$ when $A$ is a trigger event.

1. A before $B: A e<B b$.

2. A meets $B: A e=B b$ and $A b<B b$

3. A overlaps $\mathrm{B}: \mathrm{Ab}<\mathrm{Bb}$ and $\mathrm{Bb}<\mathrm{Ae}$ and $\mathrm{Ae}<\mathrm{Be}$

4. A starts $B: A b=B b$ and $\mathrm{Ae}<\mathrm{Be}$

5. A during $B: B b<A b$ and $A e<B e$

6. A finishes $B: A e=B e$ and $B b<A b$

7. A equals $B$ (coincident): $A b=B b$ and

$\mathrm{Ae}=\mathrm{Be}$ 
The above relations are qualitative, since no numerical quantity is assigned to measure the time differences. Arguing with these relations may not always be useful, as illustrated by the examples below.

\section{Examples}

(i) Consider the qualitative temporal logic statement:

A during $\mathrm{B}$ and $\mathrm{B}$ overlaps $\mathrm{C}$ :

This means $\mathrm{Bb}<\mathrm{Ab}$ and $\mathrm{Ae}<\mathrm{Be}$.

Also since $\mathrm{A}$ is a real event: $\mathrm{Ab}<\mathrm{Ae}$.

Since $\mathrm{B}$ overlaps $\mathrm{C}$, we have :

$\mathrm{Bb}<\mathrm{Cb}, \mathrm{Cb}<\mathrm{Be}$ and $\mathrm{Be}<\mathrm{Ce}$.

Thus we can infer: $\mathrm{Bb}<\mathrm{Cb}<\mathrm{Be}<\mathrm{Ce}$;

Also, we have: $\mathrm{Bb}<\mathrm{Ab} ; \mathrm{Ab}<\mathrm{Ae} ; \mathrm{Ae}<\mathrm{Be}<\mathrm{Ce}$.

Although we know $\mathrm{Bb}<\mathrm{Cb}$ and $\mathrm{Bb}<\mathrm{Ab}$ and also we know that $\mathrm{Ae}<\mathrm{Ce}$, we cannot compare $\mathrm{Ab}$ and $\mathrm{Cb}$.

However, $\mathrm{Ae}<\mathrm{Ce}$; this means $\mathrm{A}$ does not finish $\mathrm{C}$ and $\mathrm{A}$ is not equal to $\mathrm{C}$.

Thus $A$ can occur in any of the five following modes:

A before $\mathrm{C}$, A meets $\mathrm{C}$, A overlaps $\mathrm{C}$,A starts $\mathrm{C}$, A can be during $\mathrm{C}$.

Hence, from the statements provided we cannot resolve these cases uniquely.

(ii)Consider the qualitative temporal logic statement: A starts B and B finishes $\mathrm{C}$ This means $\mathrm{Ab}=\mathrm{Bb}$ and $\mathrm{Ae}<\mathrm{Be} ; \mathrm{Be}=\mathrm{Ce}$ and $\mathrm{Cb}<\mathrm{Bb}$; that is $\mathrm{Cb}<\mathrm{Ab}$ and $\mathrm{Ae}<\mathrm{Ce}$. Thus $\mathrm{Cb}<\mathrm{Ab}<\mathrm{Ae}<\mathrm{Ce}$; or $\mathrm{A}$ is during $\mathrm{C}$ (or $\mathrm{C}$ contains $\mathrm{A}$ ).

We can also say that if A starts B and B finishes $\mathrm{C}$, then $\mathrm{A}$ before $\mathrm{C}$ is inconsistent, since $\mathrm{Cb}$ $<$ Ae or $\mathrm{C}$ before $\mathrm{A}$.

(iii)Consider the qualitative temporal logic statement: A meets B and B overlaps C:

This means $\mathrm{Ae}=\mathrm{Bb}, \mathrm{Ab}<\mathrm{Bb}, \mathrm{Bb}<\mathrm{Cb}$ and $\mathrm{Cb}<\mathrm{Be}$ and $\mathrm{Be}<\mathrm{Ce}$;

Thus we have

$\mathrm{Ab}<\mathrm{Ae}(=\mathrm{Bb})<\mathrm{Cb}<\mathrm{Be}<\mathrm{Ce}$.

That is $\mathrm{Ae}<\mathrm{Cb}$ or $\mathrm{A}$ before $\mathrm{C}$.

(iv)Consider the qualitative temporal logic statement: A meets B and A starts B:

This means $\mathrm{Ae}=\mathrm{Bb}$ and $\mathrm{Ab}=\mathrm{Bb}$ or $\mathrm{Ab}=\mathrm{Ae}$. Thus $\mathrm{A}$ is just a trigger event.

(v)Consider the qualitative temporal logic statement: A before B and A during B.

That is $\mathrm{Ab}<\mathrm{Ae}$ and $\mathrm{Ae}<\mathrm{Bb}$; and $\mathrm{Bb}<\mathrm{Ab} ; \mathrm{Ae}<\mathrm{Be}$.

This means, $\mathrm{Ab}<\mathrm{Bb}<\mathrm{Ab}$ or $\mathrm{Ab}<\mathrm{Ab}$; this is inconsistent.

In order to be useful for practical application in multi-sensory perception we need to use a quantitative approach.

\section{QUANTITATIVE CONSISTENCY}

In the qualitative relations, since we have defined $<$ and $=$, we can use conventional integer arithmetic to convert them to quantitative reasoning by using addition and subtraction of integers representing time steps.

For example if $\mathrm{x}=\mathrm{y}-\mathrm{k}$ where $\mathrm{k}$ is a positive integer representing basic time units, then we can write, $\mathrm{x}<\mathrm{y}$; i.e $\mathrm{x}$ is $\mathrm{k}$ units of time before $\mathrm{y}$ or $\mathrm{x}+\mathrm{k}=\mathrm{y}$. We say that two events are coincident, if they use the same clock and the events $\mathrm{x}$ and $\mathrm{y}$ occur simultaneously. This means the shortest time difference between these two events $\mathrm{x}$ and $\mathrm{y}$ is Zero or $\mathrm{x}<\mathrm{y}$ and $\mathrm{y}<\mathrm{x}$; that is $\mathrm{x}$ and $\mathrm{y}$ are coincident. In fact, the composition, and disjunction among the relations (including 
ternary and n-ary relations) can be dealt with using the arithmetic of inequalities and the directed graph approach, to be described below.

We can now move on to quantitative temporal consistency through this example.

If we assume that the duration of $\mathrm{A}$ is $\mathrm{x}$ positive units of time, and $\mathrm{B}$ starts after $\mathrm{y}$ positive units of time and ends after $z$ positive units of time then $A b+x=A e ; A e+y=B b, B b+z=B e$.

This means $\mathrm{Ab}+\mathrm{x}+\mathrm{y}=\mathrm{Bb}$;

A during $\mathrm{B}$ means $\mathrm{Bb}<\mathrm{Ab}$ or $\mathrm{Bb}$ occurs at least one unit of time earlier than $\mathrm{Ab}$.

That is $\mathrm{Bb}=\mathrm{Ab}-1$; . Combining the equations we find that $\mathrm{Ab}+\mathrm{x}+\mathrm{y}=\mathrm{Ab}-1$ or

$\mathrm{x}+\mathrm{y}=-1$; this is impossible since $\mathrm{x}$ and $\mathrm{y}$ are positive. Hence the statement is inconsistent.

We now introduce numerical measures in the qualitative temporal relations to convert them to quantitative temporal relations. We assume that all the events described below are trigger events and time difference between the occurrence of these two events $E(i)$ and E(j) satisfy the inequality of the form: $\mathrm{a} \leq \mathrm{E}(\mathrm{i})-\mathrm{E}(\mathrm{j}) \leq \mathrm{b}$, where and $\mathrm{b}$ are non-negative integers. We use the following notation to map the positive integer inequalities among the events into a directed graph. Note that $a$ and $b$ can be zero for coincident events, and $a=b$, if there is no slackness in the constraint that is when the events occur with exact time difference.

Given any two events there is only one constraint, namely: $L(i, j) \leq E(j)-E(i) \leq U(i, j)$.

Here $\mathrm{L}(\mathrm{i}, \mathrm{j})$ and $\mathrm{U}(\mathrm{i}, \mathrm{j})$ are non-negative integers denoting lower and upper limits. The subtraction sign indicates obviously, $E(j)$ is later than $E(i)$ or equivalently, $E(i)$ is earlier than $E(j)$, thus satisfying the requirement that the time flows forward.

The above inequality can be split into two one-sided inequalities:

$E(j)-E(i) \leq U(i, j)$ and $E(i)-E(j) \leq-L(i, j)$

We now map the inequality to a directed graph (digraph) thus: the lower limit L(i,j) appears as an edge with a negative weight along the $\mathrm{j}$-i direction and the upper limit $\mathrm{U}(\mathrm{i}, \mathrm{j})$ appears as a directed edge with a positive weight along the $i-j$ direction, as shown in Figure 1. Note that $\mathrm{U}(\mathrm{i}, \mathrm{j})-\mathrm{L}(\mathrm{i}, \mathrm{j}) \geq 0$. Also, $\mathrm{U}(\mathrm{i}, \mathrm{j})=\mathrm{L}(\mathrm{i}, \mathrm{j})$ if the events have exactly specified time differences and $\mathrm{U}(\mathrm{i}, \mathrm{j})=\mathrm{L}(\mathrm{i}, \mathrm{j})=0$ when the events are coincident.

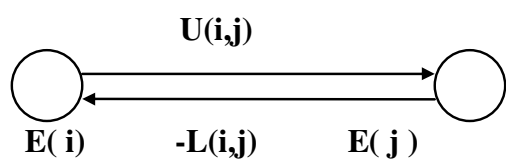

\section{Figure 1: Mapping Inequality to a Digraph}

Hence, if there are $n$ events $[i=0,1, \ldots,(n-1)]$, then we will have $n(n-1) / 2$ directed edges in the graph each representing one side of an inequality. The adjacency matrix of this directed graph that represents the simple constraint problem will have $[n+n(n-1) / 2]$ or $n(n+1) / 2$ entries in which $n$ of the entries corresponding to $E(i, i)=0$.

Further, note that the least time path between two connected nodes $\mathrm{i}$ and $\mathrm{j}$ representing event $E(i)$ and $E(j)$ respectively, is given by:

Time $(0, \mathrm{j}) \leq$ Time $(0, \mathrm{i})+\mathrm{U}(\mathrm{i}, \mathrm{j})$.

We now state and prove a variant of a theorem, proved by Shostak [16], in the context of linear inequalities.

\section{Theorem 1}

A directed graph that describes a single temporal constraint at each edge represents a temporally consistent problem, if and only if, the sum of distances across a cycle is nonnegative.

Proof: 
If part: Observe that the time elements are well founded-this means time units cannot be created or destroyed and time moves forward. So, if the problem is consistent then it implies that, they satisfy the no-decreasing condition across a cycle from the starting node of the cycle;

or

$\mathrm{E}(\mathrm{k})-\mathrm{E}(\mathrm{k}) \geq 0$. (Note that this forbids time-travel and avoids time-travel-paradox, i.e., starting from a point, travelling out and reaching the same point, earlier in time).

Only if part: If the cycle sum is negative it implies that across a cycle $\mathrm{E}(\mathrm{k})-\mathrm{E}(\mathrm{k})<0$; this implies $\mathrm{E}(\mathrm{k})<\mathrm{E}(\mathrm{k})$ which is not true since $\mathrm{E}(\mathrm{k})$ is non-decreasing with respect to itself and at the worst :

$\mathrm{E}(\mathrm{k})=\mathrm{E}(\mathrm{k})$ and the cycle sum is zero. Further, if there are no negative cycles, it is a consistent problem and the least-time path between any two nodes is well - defined. This can be obtained using the shortest path algorithm for spatial distances. This is because, for any pair of connected nodes $i$ and $j$, the least time path satisfies:

Time $(0, \mathrm{j}) \leq$ Time $(0, \mathrm{i})+\mathrm{U}(\mathrm{i}, \mathrm{j})$.

\section{Remark}

If $U(i, j)=L(i, j)$ for all $i, j$ then all the inequalities become equalities and the cycle sums are zero in both directions, for consistent problems.

\section{Example}

A simple illustration of this theorem is in the creation of the International date- line for setting up consistency of the local time of clocks having a well-defined time difference, at different places across the globe. To the left of the date-line, in clockwise direction we add +24 hours to denote next day, and to the right of the international date line in the anticlockwise direction we subtract 24 hours to denote the previous day to the travelling clock so that the cycle sum is zero either way, and the travelling clock is synchronized with the local clock, as shown below in Figure 2. Note that the time differences are exact here and we have equalities between different nodes.

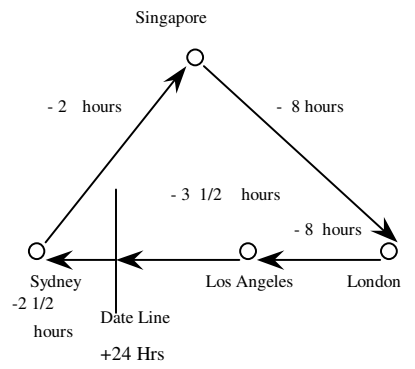

Figure 2. Consistency of International Clock

\section{USE OF COOPERATING AGENTS}

A cooperating multi-agent system can be defined as a loosely coupled network of agents that interact among them and through the environment to solve a problem Woolridge [20]. Operationally, the multiagent system carries out distributed computation by sending, receiving, handshaking and acknowledging messages and performing some local computations and has the following features:

1. An agent can carry out elementary computations and it knows its neighbor's names and other neighborhood connectivity information.

2. There is a seeding (initial) agent,that initiates the solution process.

3. Each agent can be active or inactive.

4. Initially all agents are inactive except for a specified seeding agent that initiates the computation. 
5. An active agent can do local computation, send and receive messages and can spontaneously become inactive.

6. An inactive agent becomes active, if and only if, it receives a message.

7. Each agent may retain its current belief or revise its belief as a result of receiving a new message by performing a local computation. If it revises its belief, it communicates its revised state of belief to other concerned agents; else it does not revise its solution.

Thus:

1.Each agent offers only a partial solution to a problem and holds only a partial information.

2. Control and Data are decentralized

4. Computation is not necessarily synchronous.

5. The computation terminates when the solution is reached collectively and the multi-agent system is said to be self-stabilizing.

We now describe how several agents cooperate in checking the global temporal consistency of a temporal constraint problem. For this purpose, we consider the problem of finding a least time path between any two vertices in a directed graph whose edges have a certain assigned positive or negative costs. The vertices of this directed graph correspond to events represented by agents. Here the agents communicate directly through messages (Message passing method). Here we use the terminology similar to the Agent language KQML (Knowledge Query and Manipulation Language), designed by Finn et al. [21], consisting of the following Six primitives:

1. A Performative : A single word describing the purpose of the message, e.g., Tell, Reply

2. Identity of Sender

3. Identity of Receiver

4. Language used in Content

5 Ontology-Vocabulary: Context within which the message content is to be interpreted

6. Message content

\section{Example}

Let E0,E1,E2,E3 and E4 denote sensory events constrained by the following inequalities, in which the left and right hand sides are denoted in units of time.

(1) $10 \leq \mathrm{E} 1-\mathrm{E} 0 \leq 20$

(2) $30 \leq \mathrm{E} 2-\mathrm{E} 1 \leq 40$ or (2') $60 \leq \mathrm{E} 2-\mathrm{E} 1 \leq 70$.

(3) $20 \leq$ E4-E3 $\leq 30$ or (3') $40 \leq$ E4-E3 $\leq 50$.

(4) $10 \leq \mathrm{E} 2-\mathrm{E} 3 \leq 20$

(5) $60 \leq \mathrm{E} 4-\mathrm{E} 0 \leq 70$

The above inequalities are mapped into the directed graphs to describe the relationship among the events. Since there are two possible inequalities (2) \&(2') and (3) \& (3'), we need to consider four different possibilities. Thus the temporal constraints among the events can be represented by four different directed graphs.

Figures 3 and 4 represent the two out of four possible constraints, where the former represents a consistent situation, while the latter represents an inconsistent situation. We will now describe the use of cooperating agents to solve this problem.

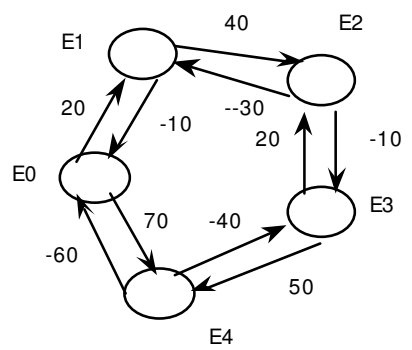

Figure 3 Temporally Consistent Problem 


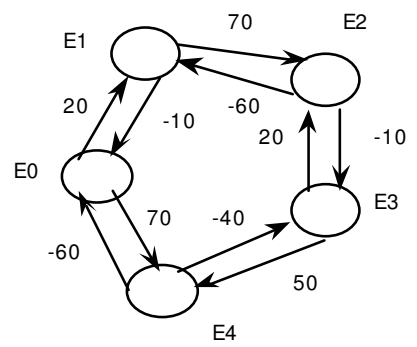

Figure 4. Temporally inconsistent Problem

\subsection{Detecting Temporal Consistency}

To detect the temporal consistency, we assume that there are $\mathrm{N}$ agents with names identical to the nodes in the graph and each agent is connected to other agents in an isomorphic manner to the given graph. Such an assumption on the topology of the network simplifies the organizational knowledge. Also each agent knows the identity of its neighbors, the direction and cost of connection of the outgoing edges. Note that the outdegree of each node is the number of sending channels and the indegree is the number of receiving channels. The production rules for multi-agent computation are as follows:

a. Initialization and update of beliefs: Agent $\mathrm{X}$ (root) sends to all its neighbors $\mathrm{Y}$ the tuple: $\mathrm{X}$, $\mathrm{s}=0, \mathrm{Y}, \mathrm{t}=0$ ) describing the name $\mathrm{X}$ of the root, and the distance $\mathrm{s}=0$ from its source neighbor, its distance $t=0$ from root; all the neighbors of the root handshake, receive, and store it. This corresponds to the initialization of beliefs.

Each agent $\mathrm{Y}$ at a distance $\mathrm{u}$ from $\mathrm{X}$ then sends its neighbor $\mathrm{Z}$ at a distance $\mathrm{w}$ from it, the tuple $(\mathrm{Y}, \mathrm{u}, \mathrm{Z}, \mathrm{u}+\mathrm{t})$ describing its name, its distance $\mathrm{u}$ from the source neighbor, its distance $\mathrm{u}+\mathrm{t}$ from the initial node. This is the initial set of beliefs of the agents.

b. Halting: Finally, the initial node checks its cycle lengths; if any one cycle is negative, it declares that the problem is inconsistent.

\section{Example}

Consider the directed graph in Figure 3, in which the edge costs are as shown; we denote the graph by the triplet, namely, a pair of nodes $(X, Y)$ followed by the cost $\mathrm{s}$ of the edge: $(\mathrm{X}, \mathrm{Y}, \mathrm{c})$. The graph in Figure 3 is then given by, (To denote nodes, we omit the letter E and use only the suffix $i$ of Ei as the labels):

$(0,1,20) ;(1,2,40) ;(2,3,-10) ;(3,4,50) ;(4,0,-60) ;(0,4,70) ;(4,3,-40) ;(3,2,20) ;(2,1,-$ $30) ;(1,0,-10)$.

We choose the vertex 0 as the root. The graph is encoded and assigned to the agents as shown in Figure 5. Here we indicate by arrows the direction of communication, and names of the communicating agents and inside the box we indicate by an ordered pair the distance of neighbour and distance of root. We apply the rules systematically.

The collective agent communication protocol and computational tree of Figure 5 is obtained from Figure 3, using the rules described. At initiation, the node labeled E0 is the root and the seeding agent. In Figure 5, we indicate by arrows the direction of communication among the agents, and an ordered quadruplet indicates: node name (the distance from neighbor, its distance to root) credit retained. The root E0 contains the ordered pair : $(0,0)$.E1 contains the pair $(20,20)$ indicating that its distance from the neighbor is 20 and from the root is 20 . Note that the constraints in Figure 5 are consistent since the cycles are non-negative the constraints in Figure 6 are inconsistent since one of the cycles is negative.

We do not know how the animal brains check for multi-sensory consistency; it should be based on very elementary logic where some kind of information circulates among the neurons. We do not claim that the brain checks for temporal consistency as in the above algorithm. 


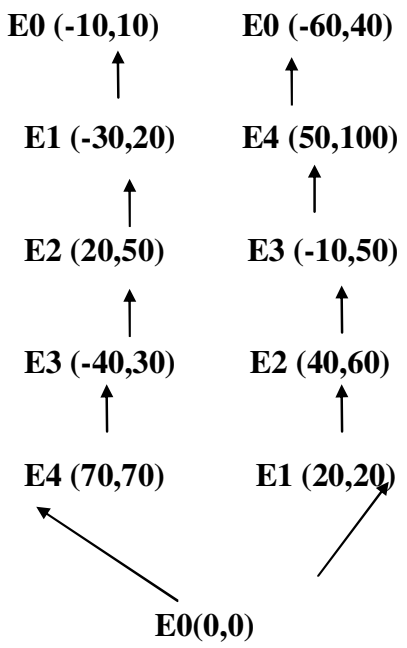

Figure 5. Agent Computation Tree

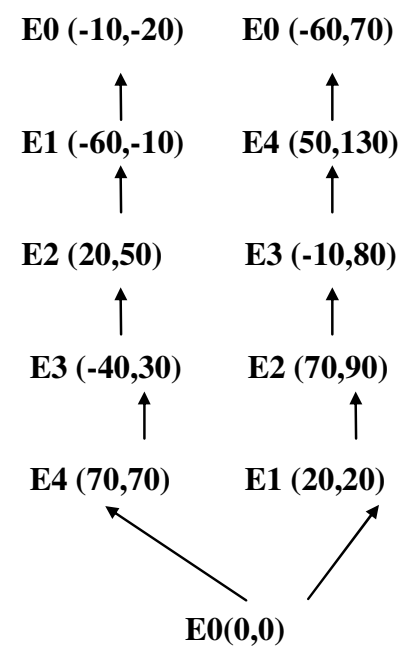

Figure. 6 Agent Computation Tree

\subsection{UML Activity Diagram}

It is convenient to visualize the above interaction among the agents using the Unified modelling language (UML) diagram, Figure 7. UML 2.0 has 13 behavioural diagrams meant specially for improved understanding Booch et al, [22] Holt, [23]. Among these diagrams the "Activity Diagram" permits a very low level modelling and is suitable for our needs, since the agent actions are at a low-level. Also it specifies the dynamic behaviour of the agents, their message and control flow and how they cooperate. Activity diagrams are made up of three basic elements: (i) Activity Node, (ii) Activity Edge, and (iii) Region.

(i) Activity Node: There are three types of activity nodes: Activity invocation, Object and control node. The Activity invocation permits us to establish traceability to the rest of the model, via operations, activities and actions.

(ii) Activity edge: This can be of two types: control flow and message or object flow.

(iii) Region: This has two main types: Interruptible activity region ands Activity partition. The former allows us to put a boundary on the diagram where activities can be interrupted; while the latter mechanism allows us to group together the different activity invocations. 


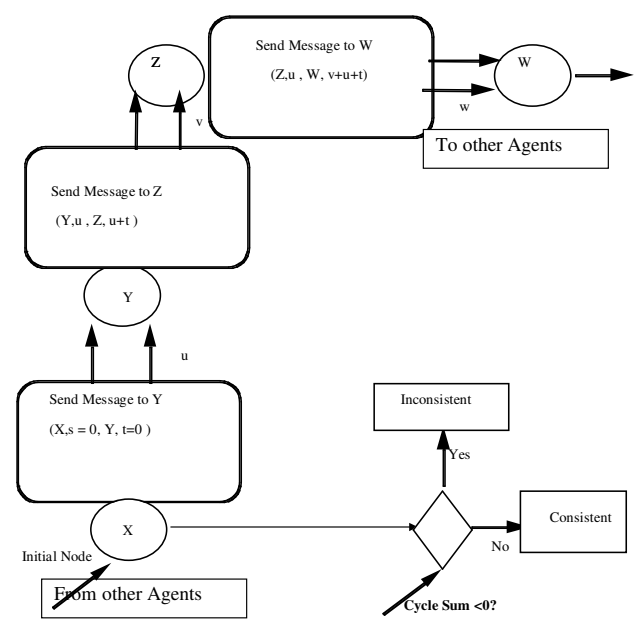

Figure 7. UML Activity Diagram

\section{CONCLUSION}

We described a cooperative agent-based algorithm to solve the simple temporal consistency problem among a set of events, using a directed graph representation. In this representation of temporal constraints among agents, each agent corresponds to an event. This algorithm is useful for several applications - in forensic science, neuro-psychology and multi-sensory information fusion- where consistency is an important criterion to check for context violations. This algorithm can enhance the potential of web -services to bring in multi-sensory reality to a scene. Numerous applications of AmI include, domestic care and supervision of the elderly, surveillance system for security, forensic sciences, and robotics.

Mobile Web 2.0 oriented next generation mobile multimedia technology will integrate collective intelligence-oriented five senses contents and provide media capabilities that can enrich the AmI environment. The integration of five senses information, along with context and temporal consistency suitably choreographed by the web-service provider and suitably orchestrated at the web service -requestor side will transform the virtual- reality to real time multi-sensory reality leading to tele-presence. Ambient Intelligence Technology (AmI) provides a new approach in using computers as a means to perform many of the human activities based on context and temporal sensory monitoring in an intelligent manner behaving like a healthy animal brain. Based on such contexts animals use the simplest form of reasoning- reflexive reasoning, rapidly, automatically and effortlessly. AmI can eventually achieve these abilities.

\section{REFERENCES}

[1]P.Remagnino., G.L.Foresti, and T.Ellis (2005) AmbientIntelligence, New York, Springer.

[2] B.J. Baars, (1997), In the theater of Consciousness, Oxford, Oxford University Press.

[3] B.J Baars,. (Ed.),(2003) Essential sources in the scientific study of consciousness, , Cambridge, Mass., MIT Press.

[4] S. Blackmore,(2006) Consciousness, Oxford, U.K., Oxford University Press.

[5] G.M Edelman, (2006)Brain science and human nature, New Haven, Conn., Yale University Press.

[6] V.K. Murthy and E.V. Krishnamurthy, in- silico consciousness: The goal of ambient intelligent computing,(2009) in UIC/ATC 2009, Brisbane, IEEE Computer Society, New York, pp. 411-416.

[7] J. de Bruijn,, ,D. Fensel, U. Keller, and R.Lara,(2005) Using the web service modelling ontology to enable semantic E-Business, Communications of the ACM, 48(12), pp.43-47. 
International Journal of Computer Networks \& Communications (IJCNC) Vol.3, No.6, November 2011

[8] A.G.Chalmers., K.Debattista , G. Mastoro-poulou. and L.P.dos Santos (2007), There-reality: Selective rendering in high fidelity virtual environments, International Journal of Virtual Reality, 6(1),pp.1-10.

[9] A.G.Chalmers D.Howard. and C. Moir (2009), Real Virtuality: A step change from Virtual Reality. in SCCG'09: Spring Conference on Computer Graphics, New York, ACM Press,2009, pp 15-22.

[10] J-H.Kim, H-J. Kwan,.and K.S.Hong, Mobile Web 2.0-oriented five senses multimedia technology with LBS-based intelligent agent,(2009), in UIC 2009, Lecture notes in Computer Science, 5585), New York, Springer Verlag, pp.281-295.

[11] J.-H,Kim , H-J.Kwan, and K.S. Hong,(2009), WiBronet-Based five senses Multimedia technology using Mobile mash-up, in ICCSA, Part II, Lecture notes in Computer Science, 5593, New York, Springer Verlag, pp.286-301.

[12] W. Hirstein, (2005),Brain Fiction, Cambridge, Mass., M.I.T. Press.

[13]G.Calvert, C. Spence and B. Stein, (2004). The Multisensory Handbook, Cambridge, Mass.,M.I.T.Press.

[14] C. Spence, and J. Driver,(2004), Crossmodal space and cross modal attention, Oxford, U.K, Oxford University Press.

[15] E.B.Goldstein, (1996), Sensation \& Perception, New York, Brooke and Cole Publishing Company.

[16] R. Shostak (1981),Deciding linear inequalities by computing loop residues, Journal of the ACM, 8 , pp.769-779.

[17] Manna,Z. \& Waldinger,R,(1993), The Deductive foundations of Computer programming, Reading, Mass., Addison Wesley.

[18] Apt, K.R (2004), Principles of Constraint Programming, Cambridge, U.K, Cambridge University Press.

[19] R. Dechter, (2003), Constraint Processing, SanFransisco, Morgan Kaufmann Publishers.

[20] Woolridge, M,(2002), An Introduction to Multi-Agent Systems, New York, John Wiley.

[21] T. Fin,(2003), KQML: An Agent communication Language , in J.M. Bradshaw (Ed) Software Agents, Cambridge, Mass., M.I.T. Press, pp. 291-316.

[22] G. Booch, J.,.Rumbaugh and I. Jacobson, (1999), The unified Modeling language User guide, Reading, Mass., Addison Wesley.

[23] J. Holt,(2004) UML for Systems Engineering, London . U,K, IEE.

\section{AUTHORS BIOGRAPHY}

Dr. V.K. Murthy is an academic visitor at the Australian National University, Canberra. His research contributions are in distributed, mobile and multimedia computing, , E-commerce, multi-agent technology and software engineering.

Address: Computer Sciences Laboratory, Research school of Information Sciences and Engineering, Bldg.115, Australian National University, Canberra, ACT 0200, Australia.

Email: edayathuk@gmail.com

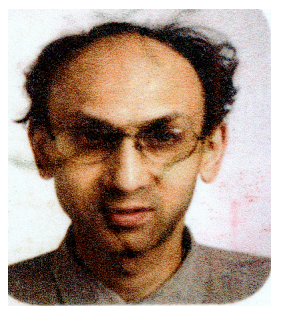

Professor E.V. Krishnamurthy is with the Computer Sciences Laboratory, Australian National University. He is the author of several books and papers in Computer Science and Information technology.

Address:

Computer Sciences Laboratory, Research school of Information Sciences and Engineering,Bldg.115 Australian National University, Canberra, ACT 0200, Australia.

Email: Evk.Krishnamurthy@anu.edu.au

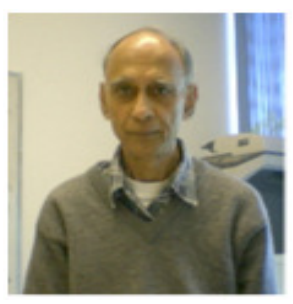

